\title{
Heat transfer enhancement of inclined projected winglet pair vortex generators with protrusions
}

\author{
Mohammad Oneissi $^{\mathrm{a}, \mathrm{b}, \mathrm{c}}$, Charbel Habchi ${ }^{\mathrm{d}}$, Serge Russeil ${ }^{\mathrm{a}}$, Thierry Lemenand ${ }^{\mathrm{b}, *}$, Daniel Bougeard ${ }^{\mathrm{a}}$ \\ a Mines Douai, EI, F59500, Douai, France \\ ${ }^{\mathrm{b}}$ Angers University, ISTIA, LARIS EA 7315, Angers, France \\ ${ }^{\mathrm{c}}$ Lebanese International University LIU, Mechanical Engineering Dept., Beirut, Lebanon \\ ${ }^{\mathrm{d}}$ Notre Dame University-Louaize NDU, Thermofluids Research Group, Zouk Mosbeh, Lebanon
}

\section{A R T I C L E I N F O}

\section{Keywords:}

Heat transfer enhancement

Winglet pair

Vortex generator

Protrusions

Plate-fin heat exchanger

Numerical simulation

\begin{abstract}
A B S T R A C T
Heat transfer enhancement in parallel plate-fin heat exchanger is examined by performing three-dimensional numerical simulations of longitudinal vortex generators (VG) with protrusions. The turbulence is modeled using the shear-stress transport (SST) $\kappa$ - $\omega$ model and validated with correlations and experimental data at Reynolds number equal to 4600. Hemi-spherical protrusions are inserted downstream two VG configurations: delta winglet type (DWP) and a new VG configuration named inclined projected winglet pair (IPWP), in various locations, leading to the definition of six different configurations. Based on the streamwise distribution of Nusselt number and friction coefficient criteria in addition to vorticity, the local performance is analyzed. Some VGs with protrusions are examined and show better performance relative to VGs standing alone. The present study highlights the different mechanisms involved in the convective heat transfer intensification by generating multiple interacting vortices while adding protrusions with low pressure drop penalty. Finally, it is found that the IPWP with protrusions, set downstream in the middle, bestows the best global performance with about $7.1 \%$ heat transfer enhancement compared to DWP configuration.
\end{abstract}

\section{Introduction}

Flow structure characteristics are fundamental for heat transfer augmentation in parallel plate-fin heat exchanger. In fact, the amount of surface heat transfer augmentation is controlled by the topology of secondary flows, three-dimensionality, shear-layer reattachment and turbulence transport induced by the devices employed on the walls of the internal passages. These devices may include cylindrical tubes [1,2], transverse vortex generators (TVGs) [3], longitudinal vortex generators (LVGs) [4,5], plane or curved VGs [6], dimples or protrusions [7], or even a combination of the above types [8,9].

These components are used in various industrial fields including electronics cooling, micro and macro-scale heat exchangers, combustion chambers and chemical reactors. This paper focuses on the addition of hemi-spherical protrusions downstream two types of LVGs, and on their capability in heat transfer enhancement caused by the generation of strongly interacting large scale longitudinal vortices. This heat transfer intensification results from the combination of three main mechanisms of heat transfer enhancement: the reduction of the laminar sub layer thickness near the wall, the development of three-dimensional turbulent layers and the swirl movement of the streamwise vortex that enhances the convective transfer, as described by Tiggelbeck et al. [10].

Protrusions downstream VGs show a significant effect on the heat transfer process since by disturbing the thin boundary layer at the wall, thus increasing the local Nusselt number as shown by Habchi et al. [11]. In fact, the addition of hemispherical protrusions between the vortex generator arrays greatly enhances the heat transfer with only a small increase in pressure drop [11]. This increase in local heat transfer is caused by increasing the temperature gradients and vorticity very close to the heated wall.

Ligrani et al. [12] reported that stronger secondary flows are present over a much larger portion of the channel cross section when protrusions are added. Secondary flow generated by protrusions enhances greatly the heat transfer process. Hwang et al. [13] explained how a dimple-protrusion patterned wall affects the heat transfer characteristics. Xie et al. [14], Li et al. [15] and Sangtarash et al. [16] showed in their investigation of flow and heat transfer in rectangular channel with dimple-protrusion geometry, that protrusions exhibit greater thermal enhancement with higher friction cost than dimples. Chen et al. [17] concluded that larger height of dimple-protrusion

\footnotetext{
* Corresponding author.

E-mail address: thierry.lemenand@univ-angers.fr (T. Lemenand).
} 


\begin{tabular}{|c|c|}
\hline \multicolumn{2}{|c|}{ Nomenclature } \\
\hline$A$ & Cross-sectional area, $m^{2}$ \\
\hline$A_{f}$ & Fin area, $m^{2}$ \\
\hline$B$ & Channel width, $m$ \\
\hline$C_{p}$ & Specific heat at constant pressure, $J \mathrm{~kg}^{-1} \mathrm{~K}^{-1}$ \\
\hline$D_{h}$ & Hydraulic diameter, $m$ \\
\hline$f$ & Friction factor \\
\hline$H$ & Channel height, $m$ \\
\hline$h$ & Convective heat transfer coefficient, $W \mathrm{~m}^{-2} \mathrm{~K}^{-}$ \\
\hline$j$ & Colburn factor \\
\hline$k$ & Thermal conductivity, $W \mathrm{~m}^{-1} \mathrm{~K}^{-1}$ \\
\hline$L$ & Channel length, $m$ \\
\hline$l$ & VG span, $m$ \\
\hline$\dot{m}$ & Mass flow rate, $\mathrm{kg} \mathrm{s}^{-1}$ \\
\hline $\mathrm{Nu}$ & Nusselt number \\
\hline$P e$ & Péclet number $=R e . P r$ \\
\hline Po & Poiseuille number \\
\hline $\operatorname{Pr}$ & Prandtl number \\
\hline$\Delta P$ & Pressure drop, $\mathrm{Pa}$ \\
\hline$q^{\prime \prime}$ & Heat flux, $W m^{-2}$ \\
\hline$R e$ & Reynolds number \\
\hline$s$ & Distance between tips of winglet pair, $m$ \\
\hline$T_{x, b}$ & Bulk temperature at position $x, K$ \\
\hline$T_{i}$ & Inlet bulk temperature, $K$ \\
\hline$T_{o}$ & Outlet bulk temperature, $K$ \\
\hline$T_{s}$ & Surface temperature, $K$ \\
\hline
\end{tabular}

\section{$U$}

$u$

$v$

$w$

$x_{v}$

$z$

\section{Greek letters}

$\mu \quad$ Dynamic viscosity, $\mathrm{Pa} s$

$\nu \quad$ Kinematic viscosity, $m^{2} s^{-1}$

$\rho \quad$ Fluid density, $\mathrm{kg} \mathrm{m}^{-3}$

\section{Abbreviations}

$\begin{array}{ll}V G & \text { Vortex Generator } \\ D W P & \text { Delta Winglet Pair } \\ R W P & \text { Rectangular Winglet Pair } \\ I P W P & \text { Inclined Projected Winglet Pair } \\ L V G & \text { Longitudinal Vortex Generator } \\ T V G & \text { Transverse Vortex Generator } \\ C F D & \text { Computational Fluid Dynamics } \\ S S T & \text { Shear-Stress Transport } \\ T E F & \text { Thermal enhancement factor } \\ P R O & \text { Protrusion } \\ H S & \text { High Spacing } \\ M & \text { Middle }\end{array}$

induces higher friction factor and Nusselt number. Bilir et al. [18] studied the optimization of the fin-tube and protrusion geometries of finned tube heat exchanger and investigated the cumulative effect of three protrusions. They concluded that the use of several protrusions provides better heat transfer performance, but increases the pressure drop, compared to the case with only one protrusion. Barik et al. [19] investigated turbulent heat transfer and fluid flow characteristics of a small rectangular channel with different protruded surfaces. An air jet impinging normal to the main flow is activated and this hybrid cooling strategy increases the pumping power in the cases with protrusions compared to that of without protrusions. The heat transfer enhancement rate is highlighted in the cases of protrusions, especially the increase with triangular protrusions is found to be more important when compared to other protrusion shapes.

The aim of the present study is to analyze the effect on the flow structure and heat transfer mechanisms of adding protrusions downstream two types of VGs. The originality of the present study lies in the way protrusions have been located downstream the VGs. In fact, their location was based on a thorough local analysis of the flow structure downstream the VG as discussed later in sections 2.2.3 and 2.2.4. Moreover, this study is performed for a novel VG designed in a previous study by Oneissi et al. [20].

The numerical method, computational domain and mesh sensitivity analysis are presented in the problem statement in section 2. Numerical validation, global performance followed by local analysis of the heat transfer phenomena and compactness comparison are discussed in section 3. Finally section 4 is dedicated to the concluding remarks.

\section{Problem description}

\subsection{Numerical model}

The flow field is governed by the three-dimensional (3D) steadystate Reynolds Averaged Navier-Stokes (RANS) equations. The continuity and momentum equations for an incompressible Newtonian fluid are: $\frac{\partial u_{i}}{\partial x_{i}}=0$

$u_{j} \frac{\partial u_{i}}{\partial x_{j}}=-\frac{1}{\rho} \frac{\partial p}{\partial x_{i}}+v \frac{\partial^{2} u_{i}}{\partial x_{j} \partial x_{j}}-\frac{\partial \overline{u_{i}^{\prime} u_{j}^{\prime}}}{\partial x_{j}}$

where the term $-\overline{u_{i}^{\prime} u_{j}^{\prime}}$ is the Reynolds stress tensor resulting from the averaging procedure on the nonlinear convective terms in the momentum equations, and the energy equation is:

$\rho c_{p} \frac{\partial T}{\partial x_{i}}=\frac{\partial}{\partial x_{i}}\left(\lambda_{e f f} \frac{\partial T}{\partial x_{i}}\right)$

where $E$ is the total energy and $\lambda_{\text {eff }}$ the effective thermal conductivity.

The solver used for the flow computation is the code ANSYS Fluent 15, which is based on an Eulerian approach to solve the Navier-Stokes equations through cell-centered finite volume discretization [11]. The code solves the conservation equations for mass and momentum in addition to the energy equation for flows involving heat transfer [21]. RANS turbulence models allow the calculation of the mean flow without first calculating the full time-dependent flow field.

For turbulent flows, $\mathrm{Re}=4600$ is used in this study, the shear-stress transport (SST) $\kappa-\omega$ model developed by Menter [22] is used. This model solves two additional partial differential equations, a modified version of the turbulence kinetic energy equation $\kappa$ used in $\kappa-\varepsilon$ model and a transport one for the specific dissipation $\omega$. Also, the shear stress transport (SST) combines the use of $\kappa$ - $\omega$ formulation in the inner parts of the boundary layer and the switching to a $\kappa-\varepsilon$ behavior in the freestream thus avoiding the $\kappa-\omega$ sensitivity to the inlet free-stream turbulence properties. In addition to that, it is characterized by its good behavior in adverse pressure gradients and separating flows while attaining accuracy and reliability [21].

The preceding attributes give the SST $\kappa-\omega$ model additional accuracy and reliability thus providing it an advantage over the standard $\kappa-\omega$ model. Moreover, the SST $\kappa-\omega$ model was used by many researchers in previous works that gave a fair matching with experimental results, as discussed by Yongsiri et al. [23] and Tang et al. [24].

This approach necessitates assessment of the wall adjacent cell size 
that insures a dimensionless wall distance $y^{+}$lower than 4, ensuring that the viscous sublayer is meshed and solved.

The SST $\kappa-\omega$ model transport equations are:

$\rho \frac{\partial}{\partial x_{i}}\left(k u_{i}\right)=\frac{\partial}{\partial x_{j}}\left(\Gamma_{k} \frac{\partial k}{\partial x_{j}}\right)+\widetilde{G}_{k}-Y_{k}$

$\rho \frac{\partial}{\partial x_{i}}\left(\omega \boldsymbol{u}_{i}\right)=\frac{\partial}{\partial \boldsymbol{x}_{j}}\left(\Gamma_{\omega} \frac{\partial \omega}{\partial \boldsymbol{x}_{\boldsymbol{j}}}\right)+\boldsymbol{G}_{\omega}-\boldsymbol{Y}_{\omega}+\boldsymbol{D}_{\omega}$

where $\widetilde{G}_{k}$ is the production of turbulence kinetic energy due to mean velocity gradients, $G_{\omega}$ is the generation of $\omega, \Gamma_{k}$ is the effective diffusivity of $\kappa\left(\Gamma_{k}=\mu+\frac{\mu_{t}}{\sigma_{k}}\right), \Gamma_{\omega}$ is the effective diffusivity of $\omega$ $\left(\Gamma_{\omega}=\mu+\frac{\mu_{t}}{\sigma_{\omega}}\right), Y_{k}$ is the dissipation of $\kappa$ due to turbulence, $Y_{\omega}$ is the dissipation of $\omega$ due to turbulence, $D_{\omega}$ is the cross-diffusion, $\sigma_{k}, \sigma_{\omega}$ are the turbulent Prandtl numbers for $\kappa$ and $\omega$ respectively, $\mu_{t}$ is the turbulent viscosity. For detailed discussion of these equations, the reader may refer to [21].

For spatial discretization of the convective terms, a scheme of double precision and second order upwind is used to consecutively solve the flow equations [25]. Central-difference and second order accuracy are selected for the diffusion terms. The coupled algorithm is used for the pressure-velocity coupling with the pseudo transient option. Pseudo transient method option is used in the present simulations, which is a form of implicit under-relaxation for steady-state cases. It allows the user to obtain solutions faster and more robustly.

\subsection{Computational domain}

\subsubsection{Boundary conditions}

The channel dimensions used for the simulations are taken from a previously designed parallel plate channel with a height $H=38.6 \mathrm{~mm}$, breadth $B=1.6 H$ and a length $L=13 H$. An isometric view showing the boundary conditions is presented in Fig. 1 .

The Reynolds number, calculated based on the hydraulic diameters $D_{h}=2 H$, is taken equal to 4600 . The reason behind selecting Reynolds equal to 4600 for this study is due to the high performance of such configuration at this Reynolds number, as shown by Oneissi et al. [20]. The hydrodynamic and thermally developing flow is computed by means of a uniform inlet velocity profile and uniform inlet temperature equal to $300 \mathrm{~K}$. Since factual simulations are the aim, realistic turbulence intensity is needed and inlet turbulence intensity equal to $3 \%$ is selected. First, it is in the range of the moderate wind tunnel intensities $(1 \%-5 \%)$, thus being realistic to experimental test benches. Second, this value for the turbulence intensity was used in recent papers [1]. The turbulence length scale, taken as a fraction of the inlet hydraulic diameter $D_{h}$, is chosen equal to $0.07 D_{h}$ as used in similar flow configurations used by Naphon et al. [26].

At the outlet, a constant pressure condition has been set with a zero gauge pressure. Isothermal walls are set for both, upper and bottom walls, with a constant temperature of $350 \mathrm{~K}$. All VGs are assumed adiabatic as the purpose is to highlight the effect of the flow structure on the convective heat transfer.

\subsubsection{DWP and IPWP configurations}

Two types of vortex generators are used in this study: Fig. 2 shows the dimensions of the classical Delta Winglet Pair (DWP) and the previously suggested Inclined Projected Winglet Pair (IPWP) vortex generators [20]. These dimensions are not for real scale heat exchanger channel, whereas they are prototypes compatible with existing wind tunnel test bench. Both configurations are implemented at an angle of attack equals to $30^{\circ}$. The same base and height dimensions are conserved for both VGs while the roll-up angle (angle between VG and lower wall) is decreased from $90^{\circ}$, for the DWP, to $30^{\circ}$ for the IPWP configuration. This geometry is obtained by the frontal projection of the DWP on a $30^{\circ}$ inclined plane from the base axis.

The goal behind this study is to improve the heat transfer of the DWP and IPWP configurations at Reynolds number equals to 4600 by inserting protrusions downstream the VG and to compare with the IPWP performance that can be found in Oneissi et al. [20].

\subsubsection{DWP with protrusion configurations}

The approach studied throughout this section is the implementation of protrusions downstream the classical DWP configuration. Scaled dimensions of protrusions consisting of hemispheres of radius equal to one fourth of the channel height is implemented and located at a distance of three times the protrusion radius downstream the VG, as in Habchi et al. [11]. Fig. 3 shows that the protrusions are located $30 \mathrm{~mm}$ downstream the VG trailing edge with a radius of $10 \mathrm{~mm}$.

Two settings of hemispherical protrusions are selected and studied in this section. The first position of the protrusion, selected in the "idle region" of the swirling flow, is identified as DWP-PRO1. A protrusion in the shade of the DWP is named DWP-PRO2, as shown in Fig. 4. Fig. 4 is the streamline cross section plane of the channel downstream the DWP alone configuration studied by Oneissi et al. [20] presented to show the location of the protrusions as a function of secondary flow pattern.

Fig. 5 shows the two configurations of DWP with protrusions: DWPPRO1 is assigned to the DWP with $62 \mathrm{~mm}$ spanwise apart protrusions which is located in an inactive region of the swirling flow, while DWPPRO2 is appointed to the DWP with $40 \mathrm{~mm}$ protrusions apart which interrupts the swirling flow of the main vortex as shown in Fig. 4. The protrusions are located $30 \mathrm{~mm}$ downstream the VG trailing edge.

\subsubsection{IPWP with protrusion configurations}

Another approach is also studied in this section by implementing a protrusion downstream the IPWP vortex generator. The same protrusions implemented to the DWP configuration are used for the IPWP geometry. Thus, the protrusions are located $30 \mathrm{~mm}$ behind the VG lower corner edge with a radius of $10 \mathrm{~mm}$. IPWP-PRO1 is assigned to the IPWP with $62 \mathrm{~mm}$ distant protrusions, while IPWP-PRO2 is appointed to the IPWP with $40 \mathrm{~mm}$ separated protrusions. Fig. 6 shows schematics of the IPWP-PRO1 and IPWP-PRO2 configurations. The protrusions are located $30 \mathrm{~mm}$ downstream the VG trailing edge.

In addition, two other geometries using protrusions of radius of $10 \mathrm{~mm}$ are conducted in this section. The first setting IPWP-HS (HS for high spacing) goes for the same as the protrusions in the IPWP-PRO1 but located in a further downstream position, located $60 \mathrm{~mm}$ downstream from the VG lower corner edge. The second setting IPWP-M (M for middle), allocates a single protrusion at the plane of symmetry of the winglet pair positioned $30 \mathrm{~mm}$ behind the VG lower corner edge. Fig. 7 shows schematics of the IPWP-HS and IPWP-M configurations.

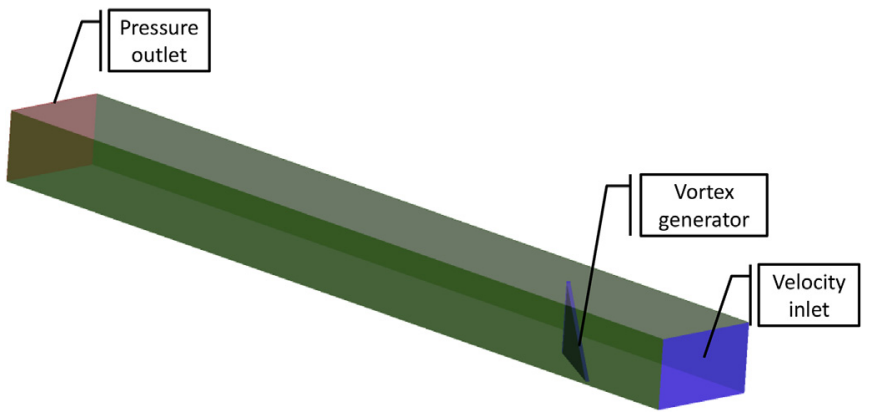

Fig. 1. Isometric view of the computational domain showing the boundary conditions: the flow is from right to left, the upper and lower surfaces are isothermal while the sides are symmetry planes. 


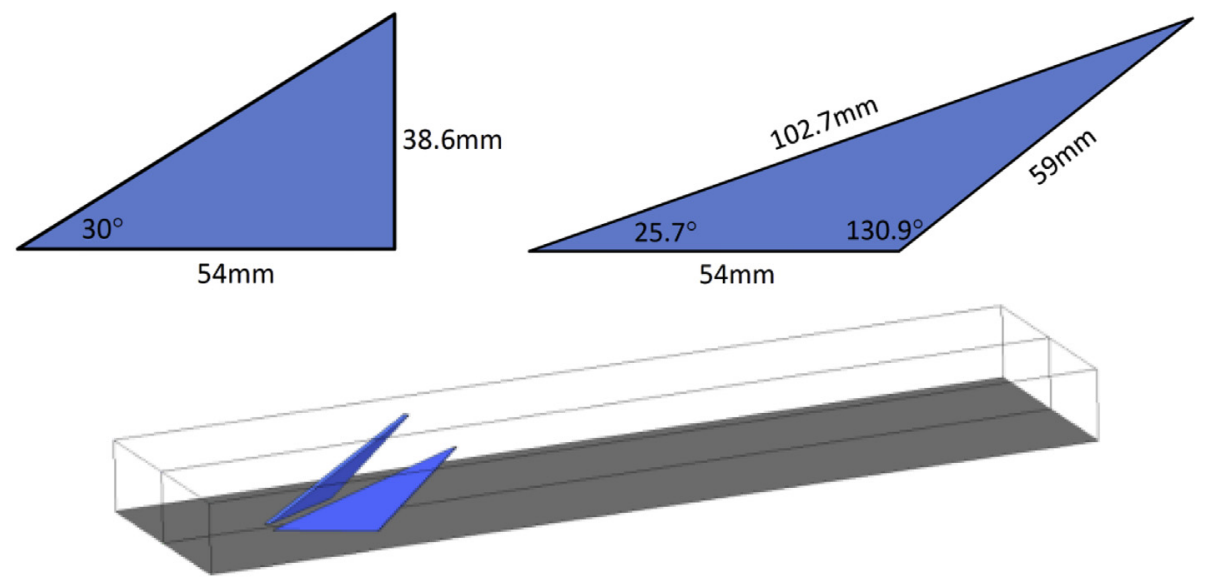

Fig. 2. (a) DWP and IPWP geometry dimensions (b) 3D view for IPWP configuration.

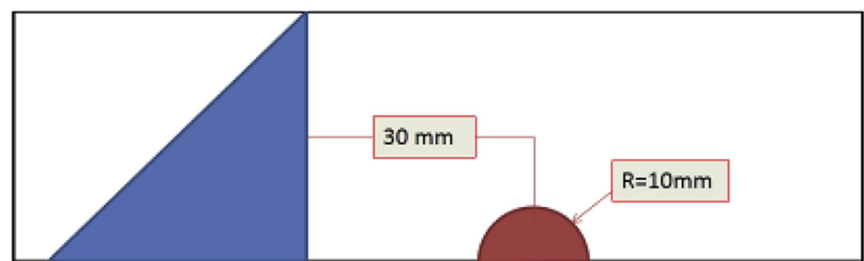

Fig. 3. Dimensions and locations of the protrusion downstream the DWP.

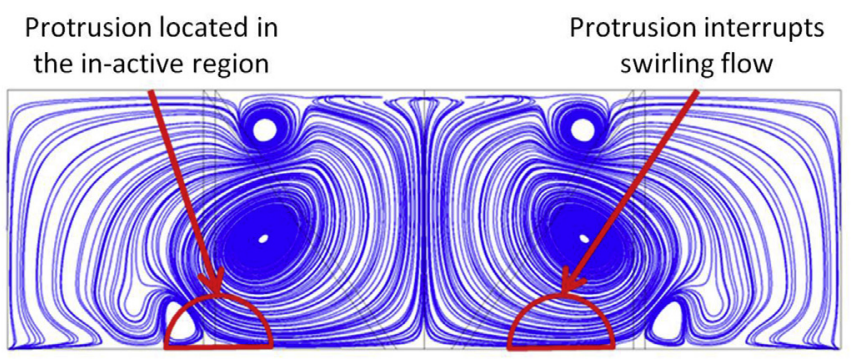

Fig. 4. DWP-PRO1 (left) and DWP-PRO2 (right) geometries, showing the protrusions positions with respect to the flow streamlines.
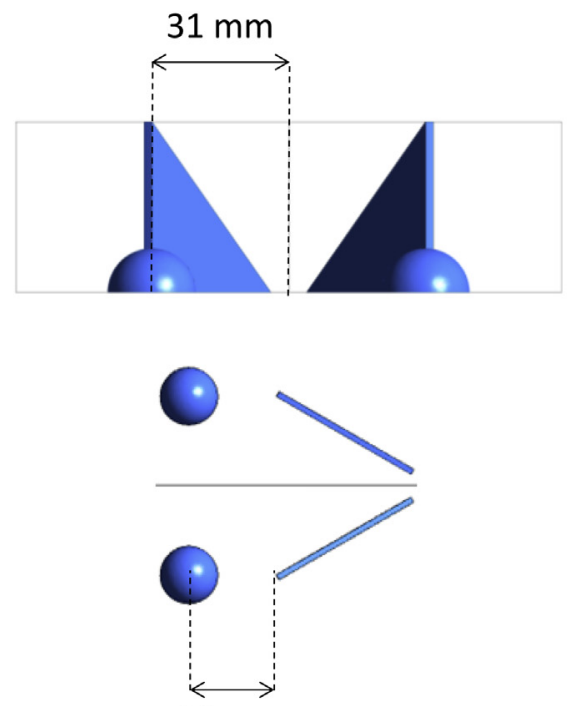

$30 \mathrm{~mm}$
Table 1 recapitulates the six different VG configurations studied.

\subsection{Mesh sensitivity study}

Non-uniform mesh with polyhedral cells is used for discretization of the computational domain. In order to maintain an accepted low value for $y^{+}$, all walls in the domain (channel and VG walls) are treated with ten inflation layers with a first layer thickness of $40 \mu \mathrm{m}$.

Mesh independency is carried out for the IPWP-PRO1 case at a Reynolds number of 4600 . Table 2 shows the different mesh densities used for the sensitivity study. In order to reach the mesh size adopted in the simulation, an arbitrary estimate of the element size is implemented, meshed and then the solution is computed. The element size is then decreased by a factor of 1.3 until the percent difference based on both global Nusselt and friction coefficient is less than $2 \%$, as described in Celik et al. [27]. The difference is calculated as follows: Difference $=\left(\overline{\mathbf{N u}}_{\text {new }}-\overline{\mathbf{N u}}_{\text {old }}\right) / \overline{\mathbf{N u}}_{\text {old }}$. Thus, as it can be observed from Table 2, mesh-3 containing more than 1,400,000 elements is chosen, since no further refinement is required because the percent difference becomes smaller than $2 \%$ after the 3 rd mesh. Mesh- 3 is then used for all the simulations. All meshes have a $y^{+}$value much lower than 5 . This was obtained by using ten inflation layers of $40 \mu \mathrm{m}$ first layer thickness
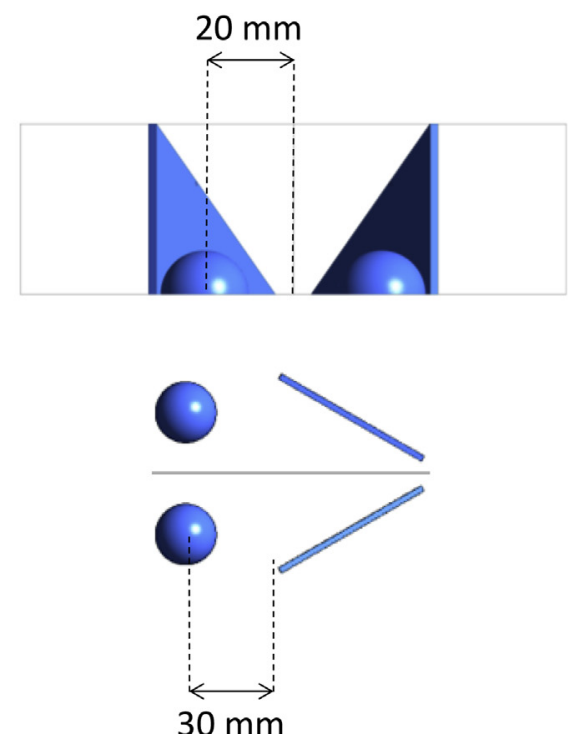

Fig. 5. Front (top) and top views (bottom) of the DWP-PRO1 (left) and DWP-PRO2 (right) geometries. 

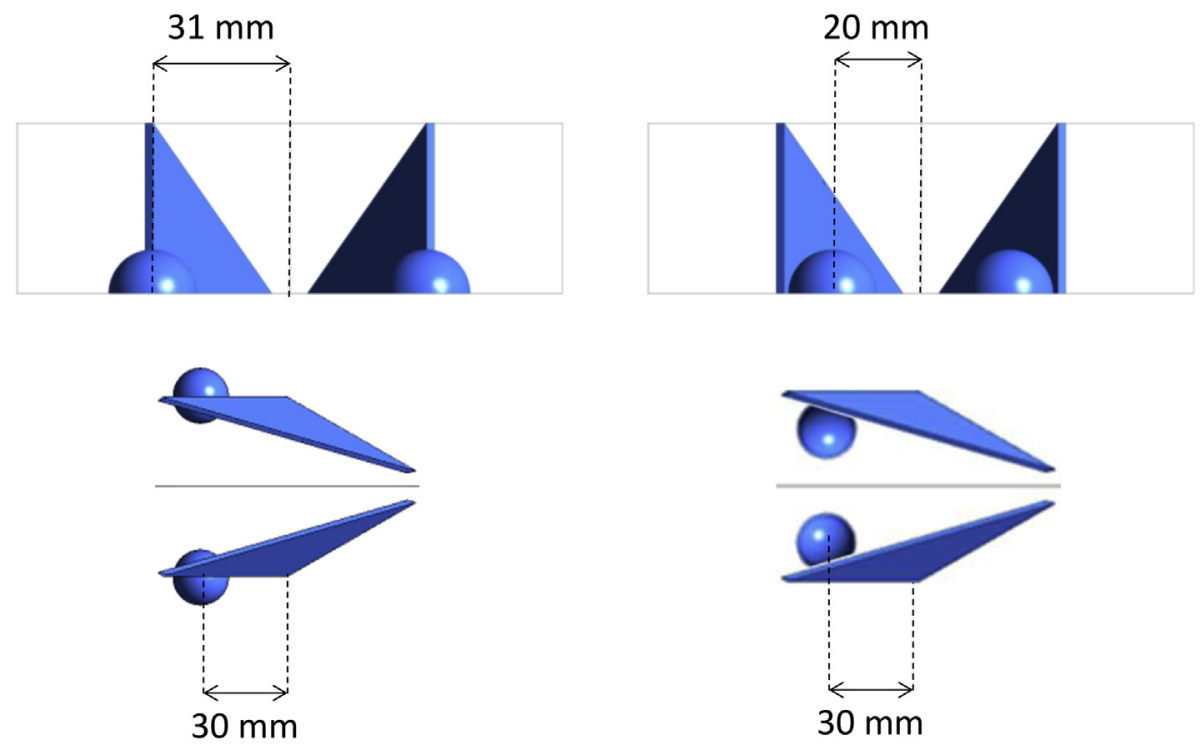

Fig. 6. IPWP-PRO1 (left) and IPWP-PRO2 (right) geometries, viewed from aft (top) and top view (bottom).

( $40 \mu \mathrm{m}-10 \mathrm{~L}$ ) which ensure that the first cell is contained in the near wall viscous sub-layer insuring no wall function is used.

\section{Results and discussion}

\subsection{Quantitative parameters and experimental validation}

The performance evaluation of the different configurations is dismantled into two main categories, global and local approaches. Global Nusselt number is given by:

$\overline{\boldsymbol{N u}}=\frac{\boldsymbol{D}_{\boldsymbol{h}} \boldsymbol{h}}{k}=\frac{2 H \boldsymbol{h}}{k}$

where $h=\frac{\dot{m} C_{p}\left(T_{o}-T_{i}\right)}{A_{f}\left(T_{s}-T_{\text {avg }}\right)}$ is obtained from the energy balance on a control volume enclosing the channel, and $T_{\text {avg }}=\frac{T_{0}+T_{i}}{2}$ is the average temperature between the inlet and outlet.

Global friction factor is obtained by:

$\boldsymbol{f}=\frac{\boldsymbol{D}_{\boldsymbol{h}} \Delta \boldsymbol{P}}{2 L \rho \boldsymbol{U}^{2}}=\frac{\boldsymbol{H} \Delta \boldsymbol{P}}{L \rho U^{2}}$
Table 1

Different VG configurations studied.

\begin{tabular}{lllll}
\hline VG & PRO1 & PRO2 & HS & M \\
\hline DWP & DWP-PRO1 & DWP-PRO2 & - & - \\
IPWP & IPWP-PRO1 & IPWP-PRO2 & IPWP-HS & IPWP-M \\
\hline
\end{tabular}

Table 2

Mesh study characteristics for the IPWP-PRO1 case.

\begin{tabular}{llll}
\hline Mesh & 1 & 2 & 3 \\
\hline Maximum element size in volume (mm) & 2.0 & 1.5 & 1.1 \\
Number of elements & 507,142 & 794,435 & $1,438,360$ \\
Inflation (mm) & $20 \mu \mathrm{m}-18 \mathrm{~L}$ & $30 \mu \mathrm{m}-12 \mathrm{~L}$ & $40 \mu \mathrm{m}-10 \mathrm{~L}$ \\
${\text { Maximum } \mathrm{y}^{+}}^{\text {Difference (\%) }}$ & 1.12 & 1.14 & 0.94 \\
& - & 2.74 & 1.18 \\
\hline
\end{tabular}

Analysis of the Nusselt number and the friction factor separately, do not give a clear idea of the global performance of the different geometries. Therefore, to compare the heat transfer efficiency of the
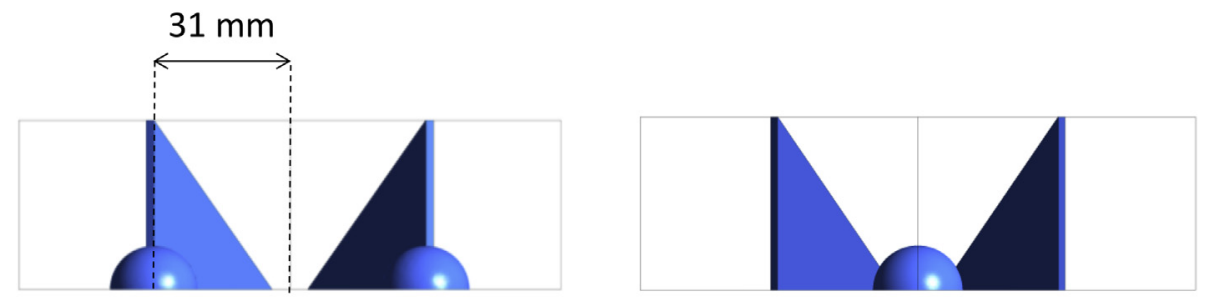

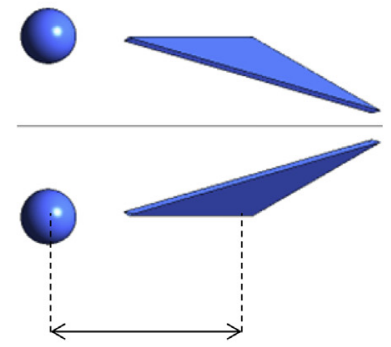

$60 \mathrm{~mm}$

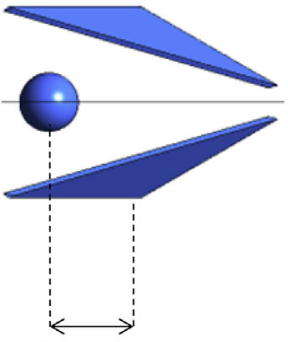

$30 \mathrm{~mm}$

Fig. 7. IPWP-HS (left) and IPWP-M (right) geometries, viewed from aft (top) and top view (bottom). 
different configurations for constant pumping power, the thermal enhancement factor (TEF) is introduced. The thermal enhancement factor is defined as the ratio of the heat exchanger (channel with VGs) convective heat transfer to that in an empty channel and represented as:

$\boldsymbol{T E F}=\frac{\boldsymbol{N u}}{\boldsymbol{N} \boldsymbol{u}_{0}}\left(\frac{f}{\boldsymbol{f}_{0}}\right)^{-1 / 3}$

where $N u_{0}$ is the global Nusselt number for empty channel, $\mathrm{Nu}$ is the global Nusselt number for channel equipped with VG, $f_{0}$ is the global friction coefficient for empty channel, $f$ is the global friction coefficient for channel equipped with VG. Therefore, the TEF which combines both the increase in Nusselt number and in pressure drop, is used to compare the different geometries studied.

Spanwise averaged local Nusselt number at a given $x$ location in the channel is given by:

$\boldsymbol{N} \boldsymbol{u}_{(x)}=\frac{\boldsymbol{D}_{h} \boldsymbol{h}_{x}}{k}=\frac{2 H q_{x}^{\prime \prime}}{k\left(T_{s}-T_{x, b}\right)}$

where $\boldsymbol{q}_{x}^{\prime \prime}$ is the heat flux at position $x$ and $T_{x, b}$ is the averaged bulk temperature in a cross sectional plane of the channel at position $x$ and defined by:

$T_{x, b}=\frac{\int_{A} \rho u C_{p} T d A}{\dot{m} C_{p}}=\frac{\int_{A} \rho u C_{p} T d A}{\rho u C_{p} d A}=\frac{1}{U A} \int_{A} u T d A$

Spanwise averaged local friction factor at a given $x$ location in the channel is given by:

$\boldsymbol{f}_{(\boldsymbol{x})}=\frac{\Delta \boldsymbol{P}}{\boldsymbol{P}_{\text {dynamic }}}=\frac{2\left(\boldsymbol{P}_{\text {in }}-\boldsymbol{P}_{x}\right)}{\rho U^{2}}$

where $\boldsymbol{U}$ is the mean flow velocity which is the average velocity at the inlet cross section.

For thermally and hydraulically developing laminar air flow, the results are compared for global Nusselt number (not the usual definition presented in equation (6)) using Stephan's correlation (see equation (13)), [28]. Stephan's correlation is used as a reference since it is the recommended analytical expression for overall Nusselt number in thermally and hydraulically laminar developing flow between two parallel-plate channels, as described by Bejan [28]. It is the integration of the local Nusselt number $\left(N u_{(x)}\right)$ over a specific length (from 0 to $x$ ) and it is defined based on the bulk temperature at each position $T_{x, b}$. This equation for global Nusselt number is valid in the range $0.1 \leq \operatorname{Pr} \leq 1,000$ for parallel plate channels.

$N u_{0-x}=\frac{1}{x} \int_{0}^{x} N u_{(x)} \cdot d x$

Stephan's correlation is defined as:

$\boldsymbol{N} \boldsymbol{u}_{0-\boldsymbol{x}}=7.55+\frac{0.024 \boldsymbol{x}^{*-1.14}}{1+0.0358 \boldsymbol{x}^{*-0.64} \boldsymbol{P r}^{0.17}}$

where

$x^{*}=\frac{x}{\operatorname{Pe} \cdot D_{h}}=\frac{x}{\operatorname{Re}_{D_{h}} . \operatorname{Pr} . D_{h}}$

Table 3 presents the global Nusselt numbers obtained from simulation compared to correlation of Stephan in laminar flow and shows a good correspondence with a maximum difference of $7.4 \%$.

Another approach is adopted to validate the numerical results in turbulent flow regime for the case with VG by comparing to Tiggelbeck's experimental results [29]. The results of interest are those dealing with a delta winglet pair (DWP) normalized to an empty channel. Tiggelbeck et al. [29] in their experiment found out that the values of the normalized global Nusselt number and friction factor for $\operatorname{Re}=4600$ are equal to 1.49 and 1.91 respectively. The same channel dimensions, boundary conditions and post-processing as Tiggelbeck's
[29] experiment are considered in the present study.

Table 4 shows a comparison between numerical simulation and experimental results obtained by Tiggelbeck et al. [29] for $\mathrm{Re}=4600$. The results are in a good agreement with each other with relatively low discrepancy, since difference between simulations and experiments is always lower than $4.7 \%$. The numerical model is reliable to predict flow and heat transfer characteristics.

Exhibited discrepancy between numerical simulations and experimental work can be mainly caused from the setup process difference between numerical simulation and experiment, in addition to data acquisition uncertainties and other factors.

\subsection{Global performance analysis of protrusion enhanced configurations}

Fig. 8 shows the performance of the two DWP protrusion enhanced configurations based on the thermal enhancement factor (TEF) criterion for Reynolds number equal to 4600 . Even though the enhancement is not firm, both DWP configurations with protrusions give better performance compared to DWP geometry that is already an interesting and enhanced configuration when compared to the smooth channel case. All comparisons are conducted relative to the DWP geometry, which is taken as a baseline configuration: DWP-PRO geometries increase the thermal enhancement factor of $2.4 \%$ and $3.2 \%$ respectively for IPWPPRO2 and IPWP-PRO1 configurations compared to the DWP geometry.

The four IPWP protrusion enhanced configurations thermal enhancement factors are presented in Fig. 9, which also plots the DWP and IPWP results. It can be noticed that both IPWP-PRO1 and IPWPPRO2 do not enhance the heat transfer compared to IPWP geometry, and IPWP-PRO1 even exhibits results lower than DWP geometry. The same PRO1 and PRO2 protrusions that increase the performances of the DWP configuration when added to DWP geometry, decreases the performances of the IPWP configuration when added to IPWP geometry. When moving the protrusions more in a downstream direction (IPWPHS) the heat transfer is then enhanced compared to the regular IPWP geometry, the augmentation of TEF is about $1.5 \%$ compared to IPWP geometry and $6.3 \%$ compared to DWP geometry. The better performance of all tested configurations is provided by IPWP-M geometry, which exhibits an augmentation of $2.3 \%$ compared to IPWP geometry and $7.1 \%$ compared to DWP geometry that shows the superiority of the IPWP-M geometry over the whole DWP and IPWP types.

Percentages of the thermal enhancement factor for all tested configurations compared to the baseline DWP geometry are listed in Table 5. Both IPWP-M and IPWP-HS provide the better TEF compared to other configurations from DWP baseline configuration. IPWP-HS can be compared to IPWP-PRO1 since the only difference is the location of the two protrusions, the distance between the VG and the protrusions is larger in the case of HS geometry. The IPWP-HS thermal enhancement factor is 1.34 compared to 1.25 for the IPWP-PRO1 case, i.e. an enhancement of $7.2 \%$ of the TEF.

IPWP-M can be compared to IPWP-PRO2 since the difference between the two configurations is the space between the protrusions, this space is decreased until zero in the case IPWP-M, i.e. there is only one protrusion centered downstream from the VG. The IPWP-M thermal enhancement factor is 1.35 compared to 1.30 for the IPWP-PRO2 case, i.e. an enhancement of $3.8 \%$ of the TEF.

Table 3

Global Nusselt number comparison between simulation and correlations.

\begin{tabular}{llll}
\hline $\begin{array}{l}\text { Reynolds } \\
\text { number }\end{array}$ & $\begin{array}{l}\text { Present } \\
\text { simulation }\end{array}$ & $\begin{array}{l}\text { Stephan's Correlation } \\
{[28]}\end{array}$ & Difference (\%) \\
\hline 270 & 7.85 & 8.42 & 6.80 \\
540 & 9.41 & 9.26 & 1.60 \\
1080 & 11.58 & 10.78 & 7.40 \\
\hline
\end{tabular}


Table 4

Normalized global Nusselt number, friction factor and thermal enhancement factor comparison between simulation and experiment results for $\mathrm{Re}=4600$.

\begin{tabular}{llll}
\hline & Simulation results & Experiment results [29] & Difference (\%) \\
\hline $\boldsymbol{N u} / \mathbf{N u} \boldsymbol{u}_{0}$ & 1.56 & 1.49 & 4.7 \\
$\boldsymbol{f} / \boldsymbol{f}_{0}$ & 1.95 & 1.91 & 2.1 \\
$\left(\mathbf{N u} / \mathbf{N u}_{0}\right)\left(\boldsymbol{f} / \boldsymbol{f}_{0}\right)^{-1 / 3}$ & 1.25 & 1.2 & 4.2
\end{tabular}

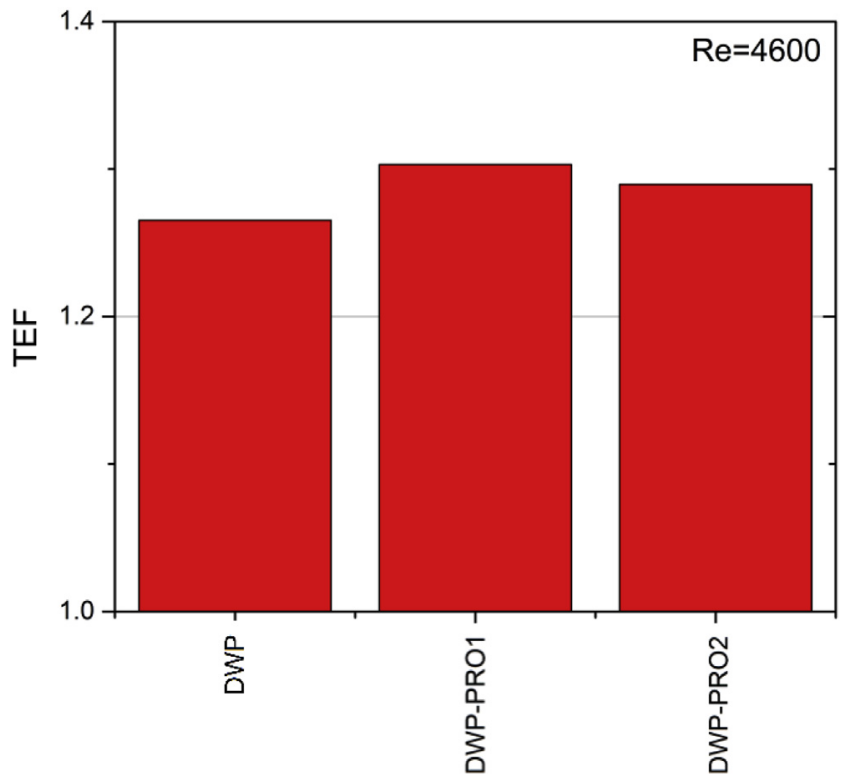

Fig. 8. Performances of the DWP and DWP-PRO geometries based on the TEF for $\operatorname{Re}=4600$.

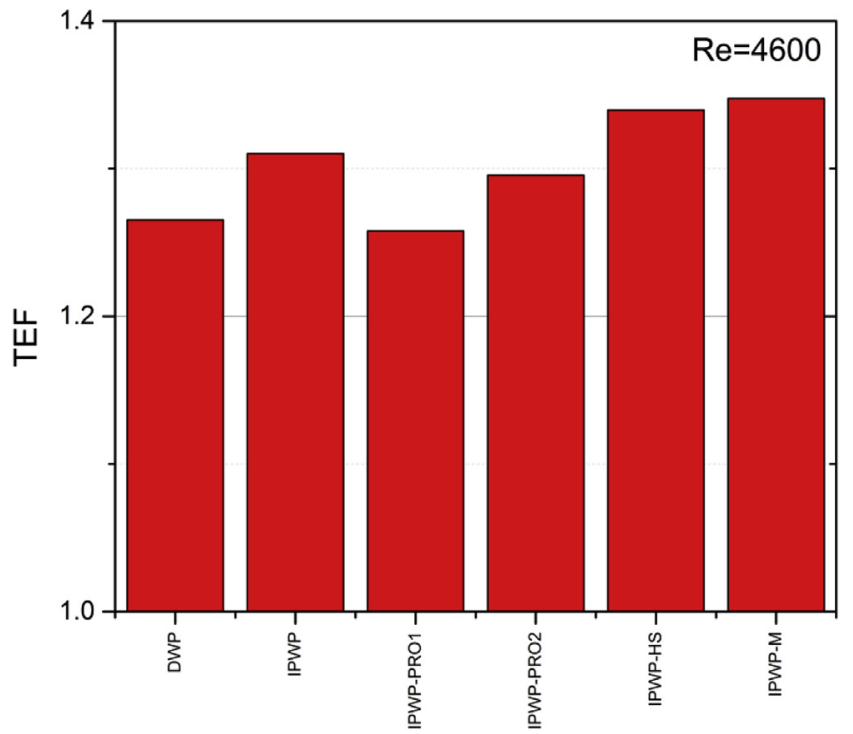

Fig. 9. Performances of the various types of IPWP based on the TEF for $\operatorname{Re}=4600$.

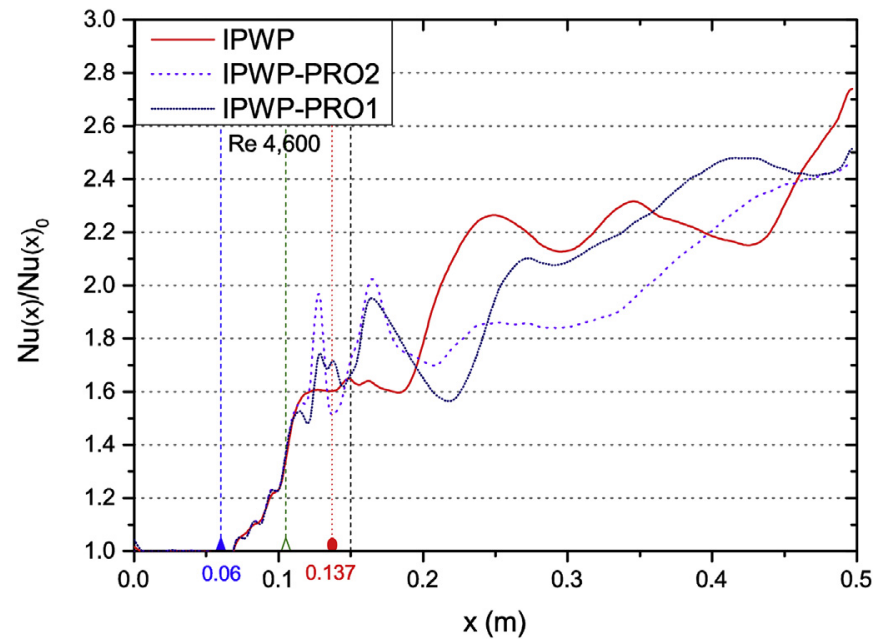

Fig. 10. Streamwise evolution of normalized spanwise averaged Nusselt number for the IPWP and IPWP-PRO1/2 configurations for $\mathrm{Re}=4600$.

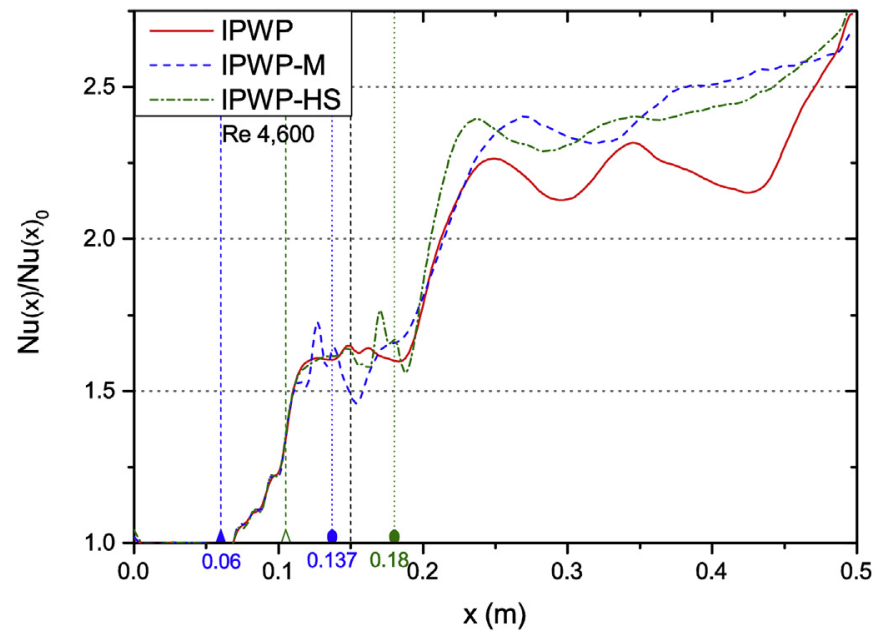

Fig. 11. Streamwise evolution of normalized spanwise averaged Nusselt number for the IPWP and IPWP-HS/M configurations for $\mathrm{Re}=4600$.

\subsection{Streamwise evolution of performance parameters}

Subsequent to demonstrating the performance of the DWP and IPWP configurations on the global scale, this section illustrates the performance from a streamwise averaged local point of view to understand the flow characteristics and the heat transfer mechanisms. The present local study is limited to the IPWP configurations due to its innovative character and improved performance compared to DWP ones. From a local scope, the performance of the IPWP with various protrusions is demonstrated throughout this section. Results for IPWP-PRO1, IPWP-PRO2, IPWP-M and IPWP-HS configurations are illustrated in Fig. 10 through Fig. 13.

Figs. 10 and 11 present $N u_{(x)} / N u_{(x)_{0}}$ the streamwise evolution of the spanwise-averaged Nusselt number throughout the channel (equation (9)), respectively for IPWP-PRO1/2 and IPWP-HS/M. Friction coefficient and vorticity are illustrated in Figs. 12 and 13 respectively. The

Table 5

Thermal enhancement fraction for all DWP and IPWP configurations studied for $\mathrm{Re}=4600$.

\begin{tabular}{|c|c|c|c|c|c|c|c|c|}
\hline & DWP & DWP-PRO1 & DWP-PRO2 & IPWP & IPWP-PRO1 & IPWP-PRO2 & IPWP-HS & IPWP-M \\
\hline TEF & 1.26 & 1.30 & 1.29 & 1.32 & 1.25 & 1.30 & 1.34 & 1.35 \\
\hline Enhancement relative to DWP (\%) & - & 3.2 & 2.4 & 4.8 & -0.8 & 3.2 & 6.3 & 7.1 \\
\hline
\end{tabular}




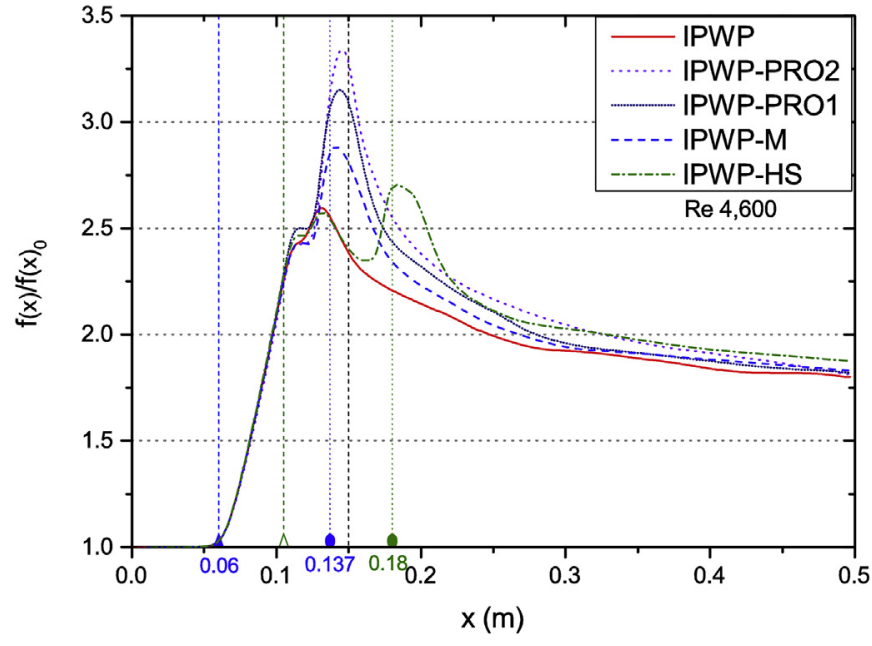

Fig. 12. Streamwise evolution of normalized spanwise averaged friction number for the IPWP and IPWP protrusion enhanced configurations for $\operatorname{Re}=4600$

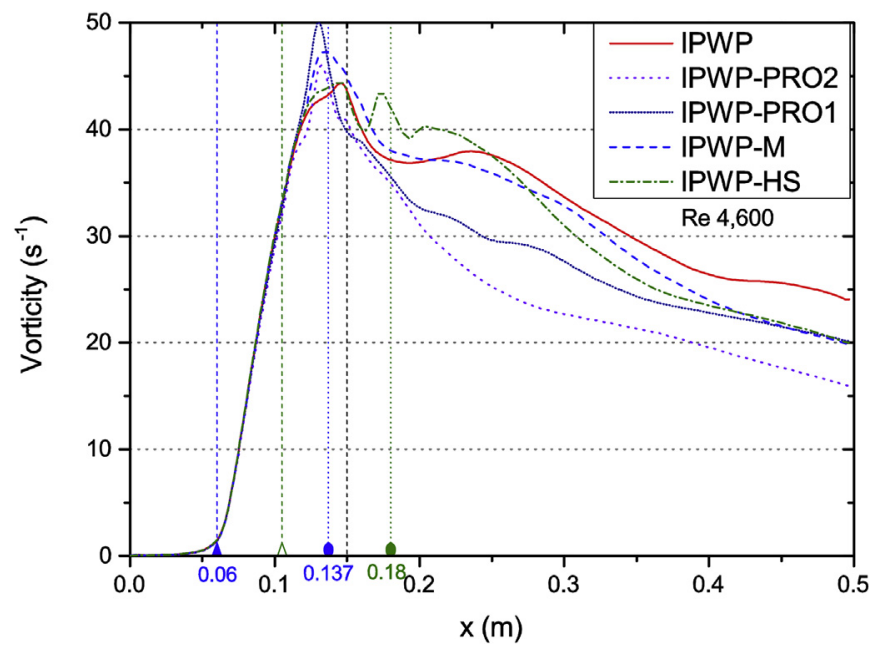

Fig. 13. Streamwise evolution of normalized vorticity for the IPWP and IPWP protrusion enhanced configurations for $\mathrm{Re}=4600$.

left vertical dotted line with a filled triangle at the bottom of the graph is the VG leading edge connected to the bottom wall. The second one (from left) designates for the separation of the bottom trailing edge with the bottom wall. The bare vertical line represents the position where the VG trailing edge merges with the upper wall. While the vertical lines with filled circle at the bottom of the graph represents the position where the protrusion is inserted at the channel bottom wall.

It can be seen from Fig. 10 that the normalized Nusselt number obtained for IPWP and IPWP-PRO1/2 configurations exhibits an increase all along the geometry but with great fluctuations. The different fluctuation behavior among perturbators is due to the interaction of the main, induced and secondary vortices. It can be seen from the figure that the normalized Nusselt number pertain a local increase due to the vortex shedding in the downstream region of the protrusions. However, it appears that IPWP-PRO1 and IPWP-PRO2 configurations do not provide any enhancement relative to IPWP baseline configuration through the channel: IPWP-PRO2 geometry gives the lowest performances and IPWP-PRO1 gives a better thermal performance compared to IPWP-PRO2. Globally the heat transfer is not increase by the presence of protrusions PRO1 and PRO2.

Fig. 11 plots $N u_{(x)} / N u_{(x)_{0}}$ the normalized spanwise Nusselt number evolution for the IPWP-HS and IPWP-M configurations compared to
IPWP reference. Again, fluctuation behavior is observed along the channel, due to the interaction of the main, induced and secondary vortices, the normalized Nusselt number pertain a local increase due to the vortex shedding in the downstream region of the protrusions. It appears that both IPWP-HS and IPWP-M increase the heat transfer versus IPWP; particularly downstream from the protrusions, the Nusselt numbers of these two geometries are always higher than that of IPWP reference.

Fig. 12 plots $f_{(x)} / f_{(x)_{0}}$ the normalized spanwise averaged friction factor (Eq. (11)) of IPWP protrusion enhanced configurations compared to IPWP case. The friction factor of IPWP-PRO1 and IPWP-PRO2 increases by $21 \%$ and $27 \%$ respectively at the peak, versus IPWP case. The reason behind a $6 \%$ difference between IPWP-PRO1 and IPWP$\mathrm{PRO} 2$ is that the protrusion in IPWP-PRO1 is located in an idle swirling flow, as previously shown in Fig. 4, and disrupt the flow downstream of VGs more strongly. Besides, the principal increase of the $f_{(x)} / f_{(x)_{0}}$ value, upstream the peak, begins from the location of the protrusion, at $x=0.137 \mathrm{~m}$. In the other hand, IPWP-HS and IPWP-M give a better result than IPWP-PRO1 and IPWP-PRO2 with an increase in the friction factor peak equal to only $4 \%$ and $9 \%$ respectively. The peak produced by the IPWP-HS configuration is moreover shifted further downstream, at $x=0.18 \mathrm{~m}$, which is located at the location of the protrusion in this case. Friction factor is locally increased for the geometries with protrusions directly downstream the protrusion: this increase is due to the base drag (or pressure drop) developed due to the flow separation over the protrusion in the downstream direction.

Vorticity of IPWP with protrusions is demonstrated in Fig. 13. Vorticity is computed over cross sectional plane area at each local location $(x)$ along the channel. Fig. 13 shows that IPWP-HS and IPWP-M configurations maintain almost the same averaged vorticity over the channel versus the IPWP geometry: it can be seen for these two enhanced cases that the vorticity increases at the protrusion location and the vorticity is then higher in the IPWP-HS/M cases compared to IPWP, however downstream from the protrusions, at about $x=0.25 \mathrm{~m}$, the vorticity of IPWP geometry becomes higher than that of IPWP-HS/M cases. IPWP-PRO1 and IPWP-PRO2 configurations present lower averaged vorticity almost over the channel versus the IPWP case. In addition, local vorticity experience similar phenomenon demonstrated previously thus increasing the vortex intensity at that region especially for the IPWP-HS/M cases.

\subsection{Flow structure}

After presenting the VG configuration with protrusions and illustrating the results from the global and local scopes, it is obvious that the IPWP with protrusions performance is noticeable. For thorough examination of the occurring convective heat transfer mechanism, crosssection planes are made visualizing various streamlines and contours in different locations downstream VGs. Fig. 14 and Fig. 15 show velocity streamlines and temperature contours respectively at different planes downstream the IPWP, IPWP-HS and IPWP-M. These planes are located at different longitudinal distances measured from the VG leading edge where $x / H$ of $\mathrm{P} 1$ to $\mathrm{P} 15$ are $2,2.5,2.7,2.9,3.1,3.3,3.5,4,4.5,5,6.5$, 8, 9, 10 and 11.5 respectively. It is shown from Fig. 14 that the same number of vortices is maintained for the three configurations at most of the channel planes, except the regions just downstream protrusions. For IPWP-HS, in the region directly downstream the protrusions (after plane P9), an additional vortex is generated due to protrusion and sustains till plane P13. Whereas the IPWP-M protrusion (located at plane P6) generates two small vortices near the bottom wall at plane P7 and dissipates rapidly before plane P9. These two vortices are formed in a form of horseshoe vortex at the protrusion base near the bottom wall.

These results are analogous to that represented in Fig. 15 that shows the effect of flow structure on the temperature distribution in these planes. For the IPWP-HS, the temperature distribution shows higher temperature zones just downstream from the protrusions (at plane 


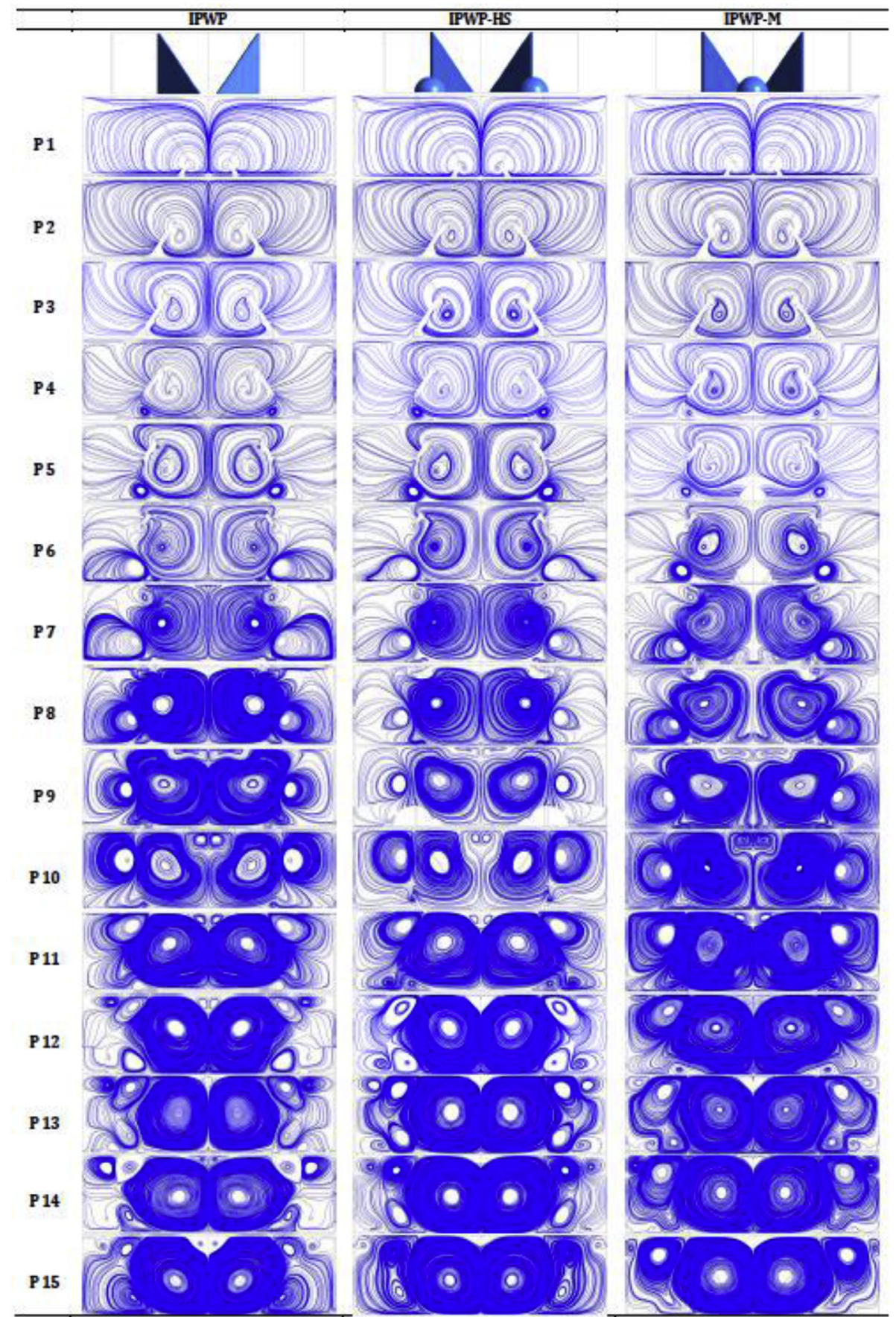

Fig. 14. Velocity streamlines for the IPWP, IPWP-HS and IPWP-M configurations for $\operatorname{Re}=4600$.

P10), which is a result of the newly generated vortices due to protrusions. The IPWP and IPWP-HS cases do not show any heat transfer distribution at the middle plane of the channel $(y / H=0)$ on the bottom wall. This is because these configurations are a common flow down configurations that direct the flow in the down direction at the channel middle plane and thus, no significant high temperatures are indicated. For the IPWP-M case, the protrusion perturbates the downwash flow by producing the small two vortices and thus increasing the heat distribution in that region (plane P7 and plane P8).

\section{Conclusions}

Three-dimensional numerical simulations of longitudinal vortex generators combined with protrusions located downstream are performed to examine heat transfer enhancement in parallel plate-fin heat exchanger. Turbulent flow with the aid of the $\kappa-\omega$ SST model is modeled and validated with correlations and experimental data at Reynolds number equal to 4600 . The aim of this work is to assess the influence of protrusions and determine if they can further increase the thermal enhancement factor of the previously published innovative vortex generator IPWP geometry.

Protrusions are first introduced to the DWP configuration resulting in the creation of the DWP-PRO1 and DWP-PRO2 geometries. Both configurations neither enhance the heat transfer nor friction factor drastically, while DWP-PRO1 having a better performance.

As well, protuberances are implemented with the IPWP geometry at different locations, leading to the production of the IPWP-PRO1, IPWPPRO2, IPWP-HS and IPWP-M configurations. These new formations end 


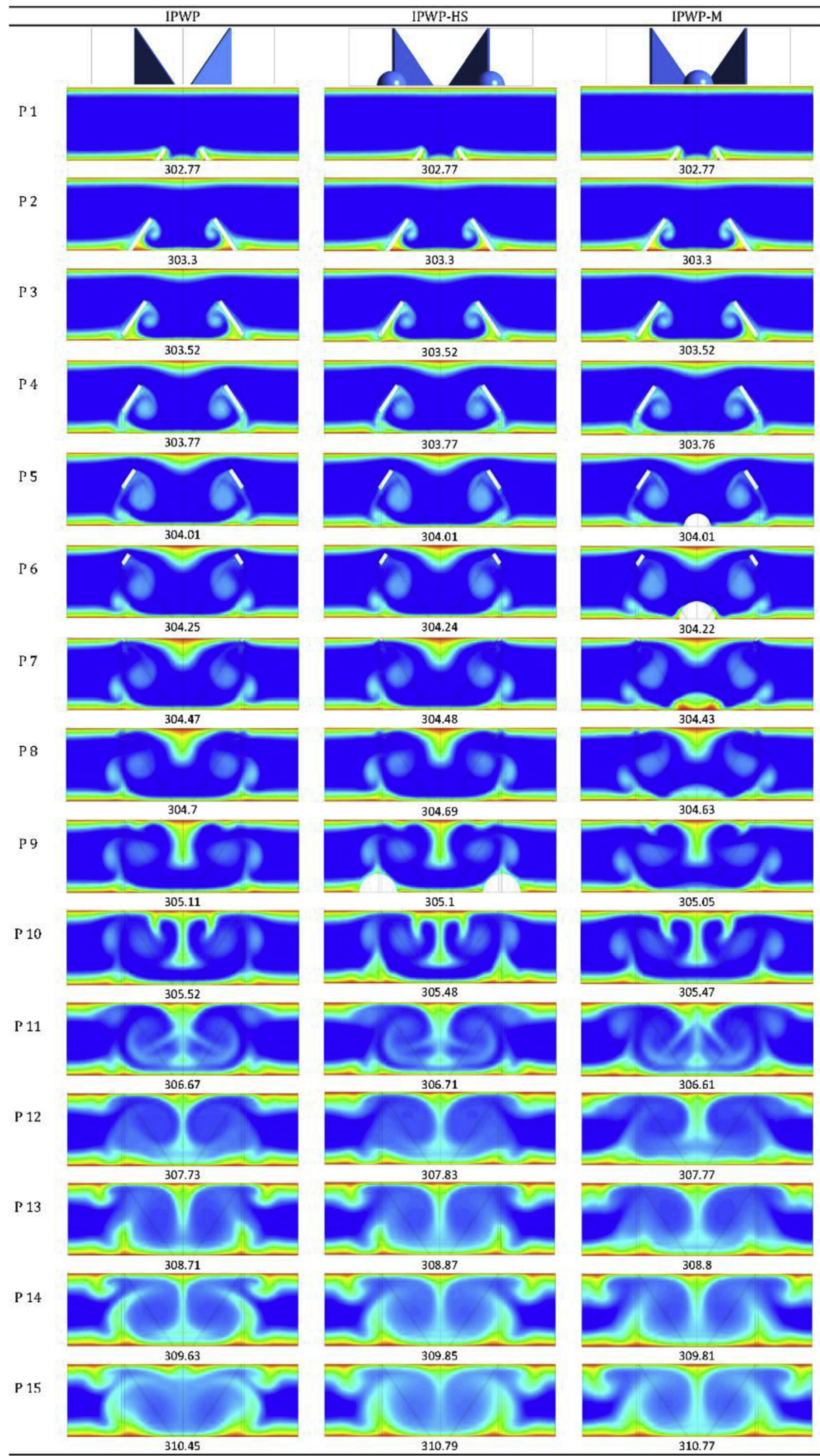

Fig. 15. Temperature contours for the IPWP, IPWP-HS and IPWP-M configurations for Re $=4600$. The area averaged temperature is indicated below each section.

up with two effects. First, an un-assisting behavior that is originated from two formations: the IPWP-PRO1 and IPWP-PRO2 configurations relative to IPWP baseline configuration, where both configurations do not outrun the regular IPWP in the total heat transfer enhancement. Second, on the other hand, the IPWP-HS and IPWP-M configurations escalate the heat transfer enhancement level. It is also revealed that the IPWP-M geometry bestow a better performance than the IPWP-HS one. Thus the present results are very promising with $7.1 \%$ heat transfer enhancement for the IPWP-M configuration compared to DWP, and $2.3 \%$ enhancement compared to IPWP geometry. Finally, delta winglet 
pair vortex generator DWP-PRO1 geometry increases the heat enhancement of $3.2 \%$ compared to DWP geometries.

\section{References}

[1] J. Voguelin Simo Tala, S. Russeil, D. Bougeard, J.-L. Harion, Numerical analysis of the fin spacing effect on the horseshoe vortex system evolution in a two-rows finned-tube heat exchanger, Int. J. Numer. Meth. Heat Fluid Flow 23 (2013) 1136-1154, https://doi.org/10.1108/HFF-03-2011-0059.

[2] A. García, J.P. Solano, P.G. Vicente, A. Viedma, The influence of artificial roughness shape on heat transfer enhancement: corrugated tubes, dimpled tubes and wire coils, Appl. Therm. Eng. 35 (2012) 196-201, https://doi.org/10.1016/j. applthermaleng.2011.10.030.

[3] M. Khoshvaght-Aliabadi, S. Zangouei, F. Hormozi, Performance of a plate-fin heat exchanger with vortex-generator channels: 3D-CFD simulation and experimental validation, Int. J. Therm. Sci. 88 (2015) 180-192, https://doi.org/10.1016/j. ijthermalsci.2014.10.001.

[4] K. Song, L. Wang, Effects of longitudinal vortex interaction on periodically developed flow and heat transfer of fin-and-tube heat exchanger, Int. J. Therm. Sci. 109 (2016) 206-216, https://doi.org/10.1016/j.ijthermalsci.2016.06.011.

[5] C. Habchi, T. Lemenand, D. Della Valle, A. Al Shaer, H. Peerhossaini, Experimental study of the turbulent field behind a perforated vortex generator, J. Appl. Mech. Tech. Phys. 56 (2015) 569-579, https://doi.org/10.1134/S0021894415040045.

[6] G. Zhou, Z. Feng, Experimental investigations of heat transfer enhancement by plane and curved winglet type vortex generators with punched holes, Int. J. Therm. Sci. 78 (2014) 26-35, https://doi.org/10.1016/j.ijthermalsci.2013.11.010.

[7] J. Lee, K.S. Lee, Correlations and shape optimization in a channel with aligned dimples and protrusions, Int. J. Heat Mass Tran. 64 (2013) 444-451, https://doi. org/10.1016/j.ijheatmasstransfer.2013.04.055.

8] H.E. Ahmed, H.A. Mohammed, M.Z. Yusoff, An overview on heat transfer augmentation using vortex generators and nanofluids: approaches and applications, Renew. Sustain. Energy Rev. 16 (2012) 5951-5993, https://doi.org/10.1016/j.rser. 2012.06.003.

[9] J.M. Wu, W.Q. Tao, Investigation on laminar convection heat transfer in fin-andtube heat exchanger in aligned arrangement with longitudinal vortex generator from the viewpoint of field synergy principle, Appl. Therm. Eng. 27 (2007) 2609-2617, https://doi.org/10.1016/j.applthermaleng.2007.01.025.

[10] S. Tiggelbeck, N. Mitra, M. Fiebig, Flow structure and heat transfer in a channel with multiple longitudinal vortex generators, Exp. Therm. Fluid Sci. 5 (1992) 425-436, https://doi.org/10.1016/0894-1777(92)90029-5.

[11] C. Habchi, S. Russeil, D. Bougeard, J.L. Harion, T. Lemenand, D. Della Valle, et al., Enhancing heat transfer in vortex generator-type multifunctional heat exchangers, Appl. Therm. Eng. 38 (2012) 14-25.

[12] P.M. Ligrani, G.I. Mahmood, J.L. Harrison, C.M. Clayton, D.L. Nelson, Flow structure and local Nusselt number variations in a channel with dimples and protrusions on opposite walls, Int. J. Heat Mass Tran. (2001), https://doi.org/10.1016/S00179310(01)00101-6.
[13] S.D. Hwang, H.G. Kwon, H.H. Cho, Heat transfer with dimple/protrusion arrays in a rectangular duct with a low Reynolds number range, Int. J. Heat Fluid Flow 29 (2008) 916-926, https://doi.org/10.1016/j.ijheatfluidflow.2008.01.004.

[14] Y. Xie, H. Qu, D. Zhang, Numerical investigation of flow and heat transfer in rectangular channel with teardrop dimple/protrusion, Int. J. Heat Mass Tran. 84 (2015) 486-496, https://doi.org/10.1016/j.ijheatmasstransfer.2015.01.055.

[15] P. Li, D. Zhang, Y. Xie, G. Xie, Flow structure and heat transfer of non-Newtonian fluids in microchannel heat sinks with dimples and protrusions, Appl. Therm. Eng. 94 (2016) 50-58, https://doi.org/10.1016/j.applthermaleng.2015.10.119.

[16] F. Sangtarash, H. Shokuhmand, Experimental and numerical investigation of the heat transfer augmentation and pressure drop in simple, dimpled and perforated dimpled louver fin banks with an in-line or staggered arrangement, Appl. Therm. Eng. 82 (2015) 194-205, https://doi.org/10.1016/j.applthermaleng.2015.02.073.

[17] Y. Chen, Y.T. Chew, B.C. Khoo, Heat transfer and flow structure on periodically dimple-protrusion patterned walls in turbulent channel flow, Int. J. Heat Mass Tran. 78 (2014) 871-882, https://doi.org/10.1016/j.ijheatmasstransfer.2014.07.036.

[18] L. Bilir, Z. Ilken, A. Erek, Numerical optimization of a fin-tube gas to liquid heat exchanger, Int. J. Therm. Sci. 52 (2012) 59-72, https://doi.org/10.1016/j. ijthermalsci.2011.09.010.

[19] A.K. Barik, A. Mukherjee, P. Patro, Heat transfer enhancement from a small rec tangular channel with different surface protrusions by a turbulent cross flow jet, Int. J. Therm. Sci. 98 (2015) 32-41, https://doi.org/10.1016/j.ijthermalsci.2015.07. 003.

[20] M. Oneissi, C. Habchi, S. Russeil, D. Bougeard, T. Lemenand, Novel design of delta winglet pair vortex generator for heat transfer enhancement, Int. J. Therm. Sci. 109 (2016), https://doi.org/10.1016/j.ijthermalsci.2016.05.025.

[21] Ansys, Turbulence models, ANSYS Fluent 12.0 Theory Guid, 2009.

[22] F.R. Menter, 2-Equation eddy-visocity turbulence models for engineering applications, AIAA J. 32 (1994) 1598-1605, https://doi.org/10.2514/3.12149.

[23] K. Yongsiri, P. Eiamsa-Ard, K. Wongcharee, S. Eiamsa-Ard, Augmented heat transfer in a turbulent channel flow with inclined detached-ribs, Case Stud. Therm. Eng. 3 (2014) 1-10, https://doi.org/10.1016/j.csite.2013.12.003.

[24] X.Y. Tang, D.S. Zhu, Flow structure and heat transfer in a narrow rectangular channel with different discrete rib arrays, Chem. Eng. Process. Process Intensif. 69 (2013) 1-14, https://doi.org/10.1016/j.cep.2013.01.005.

[25] R.F. Warming, R.M. Beam, Upwind second-order difference schemes and applications in aerodynamic flows, AIAA J. 14 (1976) 1241-1249, https://doi.org/10 $2514 / 3.61457$.

[26] P. Naphon, K. Kornkumjayrit, Numerical analysis on the fluid flow and heat transfer in the channel with V-shaped wavy lower plate, Int. Commun. Heat Mass Tran. 35 (2008) 839-843, https://doi.org/10.1016/j.icheatmasstransfer.2008.03.010.

[27] I.B. Celik, U. Ghia, P.J. Roache, C.J. Freitas, H. Coleman, P.E. Raad, Procedure for estimation and reporting of uncertainty due to discretization in CFD applications, J. Fluid Eng. 130 (2008) 78001, https://doi.org/10.1115/1.2960953.

[28] A. Bejan, Forced Convection : internal flows, Heat Transf. Handb. (2003) 395-438.

[29] S. Tiggelbeck, N.K. Mitra, M. Fiebig, Comparison of wing-type vortex generators for heat transfer enhancement in channel flows, J. Heat Tran. 116 (1994) 880-885 https://doi.org/10.1115/1.2911462. 